\title{
Facile quantification and identification for reducing gases over a wide concentration range
}

\author{
C. Schultealbert, T. Baur, A. Schütze, T. Sauerwald \\ Saarland University, Lab for Measurement Technology, Saarbrücken, Germany \\ c.schultealbert@Imt.uni-saarland.de
}

\begin{abstract}
:
Two dedicated methods for quantification and identification for metal oxide semiconductor gas sensors with a specific temperature cycled operation (TCO) are presented. Both are giving an estimate for a certain parameter of the sensor relaxation caused by a step from high to low temperature. In this relaxation a highly oxidized sensor surface is gradually reduced by gas. The relaxation parameter is evaluated by calculating the slope of the logarithmic conductance over time for small gas concentration or by an estimation of the relaxation time constant for high concentrations respectively. A linear calibration curve between the relaxation parameter and the gas concentration is found for carbon monoxide, hydrogen and ammonia in the concentration range from $10 \mathrm{ppb}$ to $100 \mathrm{ppm}$. For benzene the relaxation depends in form of a power law (exponent 0.4-0.5) with the gas concentration. The results can be interpreted within the framework of a general model for TCO MOS sensors.
\end{abstract}

Key words: MOS gas sensor, temperature cycled operation, calibration, linear, sensitivity.

\section{Introduction}

Metal oxide semiconductor (MOS) gas sensors are widely studied for many applications [1] and it is well-known that their selectivity, can be improved by the usage of temperature cycles [2]. Models of the temperature cycled operation are typically based on rate equations for the change of surface states occupancy [3]. In a previous work we could show that sensitivity and stability can be improved large by a TCO optimization along this principle [4]. Here we derive a method for the quantitative read-out of MOS gas sensors over a larger concentration range [5].

\section{Techniques}

The sensor model presented in [4] describes the conductance as a thermal activated process (grain boundary dominated), while the activation energy itself is depending on the oxidation of the surface. At high temperature phases the equilibrium tends to a stronger oxidation compared to low temperature. A sharp step from high to low temperature leads, therefore, to surface oxidation above equilibrium. The following relaxation constant (reduction of excess negative surface charge) is then linear with the concentration of reducing species on the surface and can be described with a rate constant $\mathrm{k}$ reaction process on the surface.
We use two different techniques to estimate k:

i) direct evaluation of rate constant, by fitting the slope on the low temperature phase $(10-20 \mathrm{~s}$ in Fig. 1) which is working if equilibrium is not reached during the low temperature phase and after that the

ii) T-evaluation, i.e. the time constant which is obtained via the time-stamp where the sensor signal reaches $63 \%$ of its highest value, which considering a first order process should be antiproportional to $\mathrm{k}$.

\section{Experimental Setup}

The measurements were conducted using a commercial AS-MLV-P2 sensor (ams sensor solutions, Germany). The temperature cycle consists of three high temperature phases $\left(450{ }^{\circ} \mathrm{C}, 3 \mathrm{~s}\right)$ followed each by a low temperature phase of $27 \mathrm{~s}(150,200$ and $250{ }^{\circ} \mathrm{C}$ ), which makes in total $90 \mathrm{~s}$. The sensing layer's conductance was measured via a logarithmic amplifier, so the recorded ADCvalues can directly be used for the presented techniques above. We studied carbon monoxide, hydrogen, ammonia and benzene. Concentrations obtained by dynamic dilution ranged from $10 \mathrm{ppb}$ to $100 \mathrm{ppm}$ at $50 \% \mathrm{RH}$. 


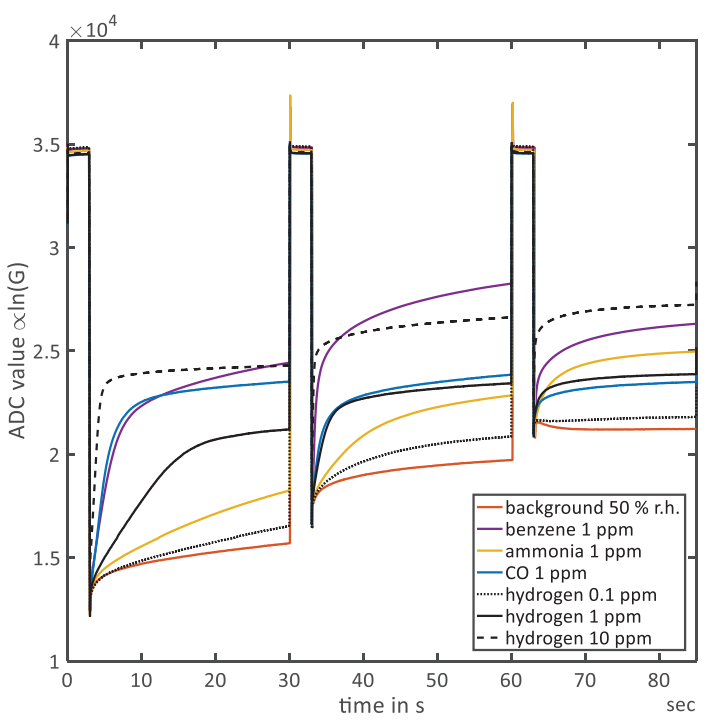

Fig. 1. Measured $A D C$ values (proportional to the logarithm of the sensor conductance) over one temperature cycle at different concentrations. The three high phases correspond to $450^{\circ} \mathrm{C}$, the three lower ones to 150,200 and $250^{\circ} \mathrm{C}$. Quantification (black lines) is based on the relaxation process during the first low temperature phase (slope and time constant) whereas identification can be achieved by comparing the relaxation on different temperature phases [5].

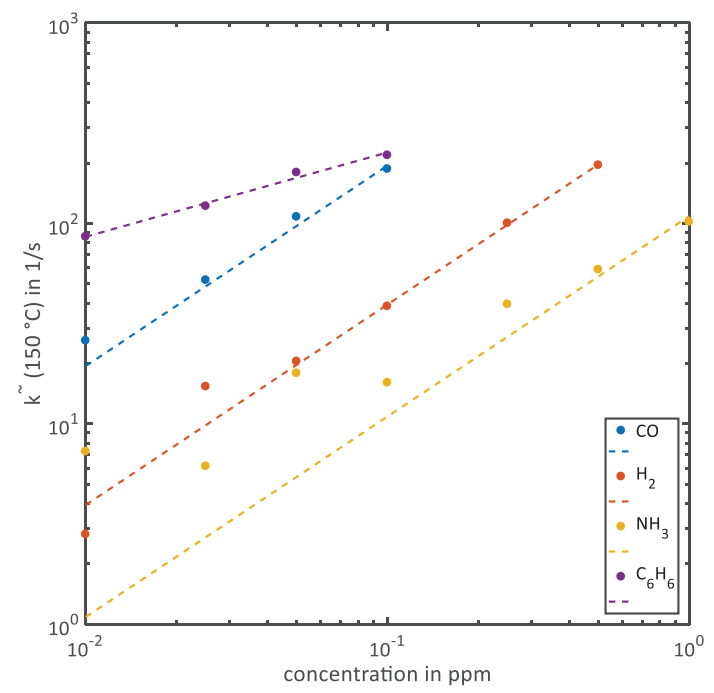

(a)

\section{Results}

In Fig. 1 the obtained raw signals for different cycles are shown. The black lines corresponding to different hydrogen concentrations indicate how the quantification works. For $0.1 \mathrm{ppm}$ equilibrium is not reached during the $150{ }^{\circ} \mathrm{C}$ phase (left), whereas for $1 \mathrm{ppm}$ a constant value is already reached after about $15 \mathrm{~s}$, for $10 \mathrm{ppm}$ after $2 \mathrm{~s}$. It can also be seen that gases can be identified for example by a linear discriminant analysis (LDA) when using the relaxation processes at different temperatures [5]. In Fig. 2 the calibration curves derived from the measurements are shown. For both techniques a linear calibration curve $a \cdot x$ for carbon monoxide, hydrogen and ammonia can be found. For benzene both methods yield a calibration curve in form of a power law $a \cdot x^{b}$.

\section{Discussion and Conclusion}

The presented physically motivated quantification method yields a calibration curve which is in accordance with the model linear, except for benzene.

A simple and general correlation between target concentration and sensor signal, how it is described here, is needed to perform proper calibrations of e.g. a batch of gas sensors.

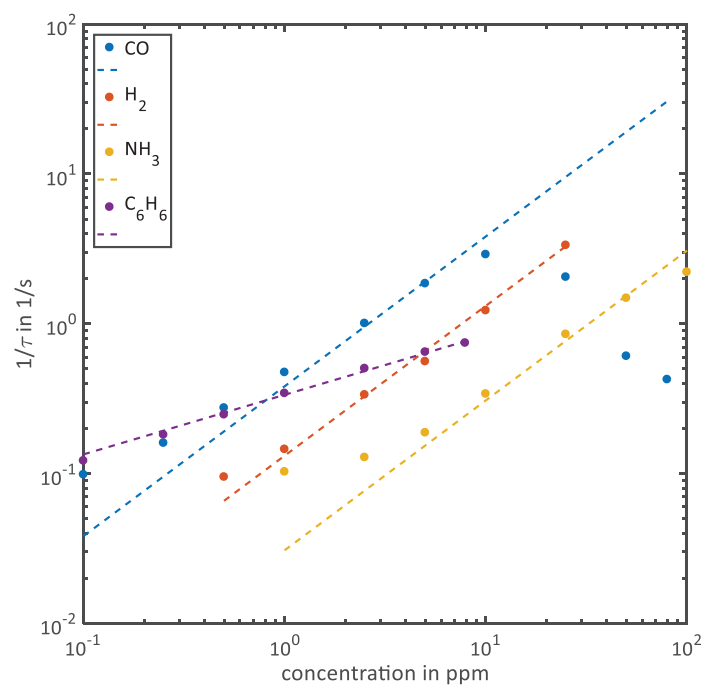

(b)

Fig.2.(a) k-evaluation for low concentrations and (b) $t$-evaluation for the higher concentrations. In both cases all gases can be assumed as a line through origin except for benzene which is represented best by a power law [5].

\section{References}

[1] D. Kohl, Journal of Physics D: Applied Physics 34, R125 (2001); doi:10.1088/0022$3727 / 34 / 19 / 201$

[2] J. Ding, T Mcavoy, RE Cavicchi, S. Semancik Sensors Actuators B Chem. 2001;77:597-613.

[3] A.P. Lee, B.J. Reedy. Sensors Actuators $B$ Chem. 60, 35-42 (1999); doi:10.1016/S09254005(99)00241-5
[4] C. Schultealbert, T. Baur, A. Schütze, T. Sauerwald, Sensors Actuators B Chem. 239, 390-396 (2017); doi:10.1016/j.snb.2016.08.002

[5] C. Schultealbert, T. Baur, A. Schütze, T. Sauerwald, Sensors 18, 744 (2018); doi:10.3390/s18030744 